International Journal of Engineering \& Technology, 7 (2) (2018) 939-947
International Journal of Engineering \& Technology
SPC
Website: www.sciencepubco.com/index.php/IJET
doi: $10.14419 /$ ijet.v7i2.10743
Research paper

\title{
An Investigation on Land Cover Mapping Capability of Classical and Fuzzy based Maximum Likelihood Classifiers
}

\author{
Shivakumar B $\mathbf{R}^{1^{*}}$ and S V Rajashekararadhya ${ }^{2}$ \\ ${ }^{1}$ NMAM Institute of Technology, Nitte, India \\ ${ }^{2}$ Kalpataru Institute of Technology, Tiptur, India \\ *Corresponding author E-mail:shivkumarbr@gmail.com
}

\begin{abstract}
In the past two decades, a significant amount of research has been conducted in the area of information extraction from heterogeneous remotely sensed (RS) datasets. However, it is arduous to exactly predict the behaviour of the classification technique employed due to issues such as the type of the dataset, resolution of the imagery, the presence of mixed pixels, and spectrally overlapping of classes. In this paper, land cover classification of the heterogeneous dataset using classical and Fuzzy based Maximum Likelihood Classifiers (MLC) is presented and compared. Three decision parameters and their significance in pixel assignment is illustrated. The presented Fuzzy based MLC uses a weighted inverse distance measure for defuzzification process. 10 pixels were randomly selected from the study area to illustrate pixel assignment for both the classifiers. The study aims at enhancing the classification accuracy of heterogeneous multispectral remote sensor data characterized by spectrally overlapping classes and mixed pixels. The study additionally aims at obtaining classification results with a confidence level of $95 \%$ with $\pm 4 \%$ error margin. Classification success rate was analysed using accuracy assessment. Fuzzy based MLC produced significantly higher classification accuracy as compared to classical MLC. The conducted research achieves the expected classification accuracy and proves to be a valuable technique for classification of heterogeneous RS multispectral imagery.
\end{abstract}

Keywords: Accuracy assessment; Fuzzy topology; Mahalanobis distance; Maximum likelihood classification; Remote sensing.

\section{Introduction}

Remotely Sensed (RS) image classification has formed itself as one of the foremost engaging technique for efficiently extracting information from RS data. A large number of environmental and socio-economic applications, such as maps, forestry, urban planning and so on, rely on the success rate of RS classification [1], [2]. Though a substantial number of researchers have bestowed their study towards remotely sensed image classification, it has still remained a challenging task within remote sensing fraternity. Hence, it has become necessary to analyse and develop new classification techniques to achieve better classification results

The overall process of RS classification can be broken down to few necessary steps; choice of an appropriate classification algorithmic rule, image preprocessing, collecting training samples, feature extraction, post-classification processing, and accuracy assessment [3]. RS image classification has been broadly classified based on the requirement of training into two types; Unsupervised and Supervised classification. Unsupervised classification techniques are most frequently used to understand the spectral characteristics of land cover classes. They produce a clustered image where similar characteristic pixels are grouped together to form a cluster [1], [3]. Supervised classification requires training data and so involves analyst's intervention to train the algorithm.

Image classification can also be categorized into Hard and Soft classification techniques based on whether the output is a definitive decision about the land cover class or not. Maximum Likelihood
Classifier (MLC) is a hard classifier based on Bayesian theorem where a pixel is considered as the basic building block of the image and is considered indecomposable. It also assumes that land cover classes have a normal distribution. A pixel under hard classification considers classical set theory where a pixel is assumed to either belong to a class or not [1]. This creates serious limitations when classifying heterogeneous datasets using classical MLC. Heterogeneous datasets are characterized by overlapping spectral classes. Pixels that represent these overlapping areas are referred to as mixed pixels or mixels, and represent more than one class perhaps due to differences due to health, age, and so on.

Training the algorithm necessitates the analyst to possess deep knowledge regarding the geographic area under consideration and its land covers [1], [3]. However, gathering ground truth details could be an elaborate and expensive process [4]. During training, an analyst is forced to collect only pure pixels from the study area as hard classifiers are insensitive to mixed pixels [5]. This affects the classification performance of MLC [1]. Hence, there is a need for a classifier that considers the fuzziness within a pixel.

The soft or Fuzzy classification has been a field of great interest in the remote sensing fraternity for the last three decades. The Fuzzy classification has been known for its capacity to extract more useful information from a heterogeneous RS data. Unlike classical MLC, it does not make any assumption on the distribution of the data and is shown to provide detailed information [6]. A large number of studies have been conducted for classifying RS data using Fuzzy logic [6]-[12]. 


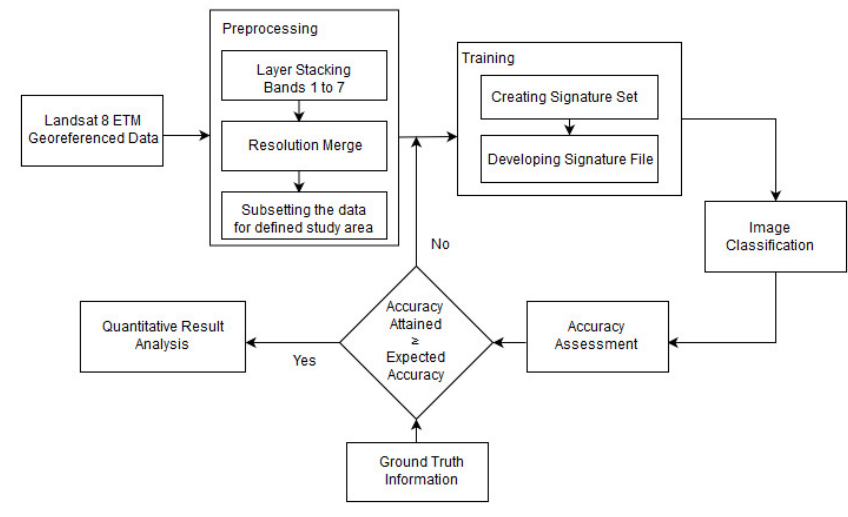

Figure 1: General flow of the methodology.

The three main stages of the Fuzzy classification are; Fuzzification of each pixel, using Fuzzy reasoning rule to assign that pixel to a class, and a defuzzification operation to obtain a hard classification map [9]. Similar to classical MLC, Fuzzy classifier also involves training phase. However, training a Fuzzy based classifier allows the use of both pure pixels and mixels [13].

The objective of this study was to perform land cover mapping of the considered study area using Fuzzy theory based MLC. It was intended to produce classification results with a confidence level of $95 \%$ with $\pm 4 \%$ error margin. Further, results obtained by employing Fuzzy theory into MLC are compared with that of the classical MLC, both qualitatively and quantitatively. Parameters that define the classification success rate are identified and presented with relevant illustrations. The overall methodology followed is illustrated by the flowchart shown in Figure 1.

The rest of the paper is organized as follows. In section 2, details about the data and study area are presented. Section 3 provides information on feature extraction and signature collection. Section 4 discusses the classification techniques employed. Section 5 provides brief information on accuracy assessment. Section 6 contains results obtained during the study. Section 7 provides the conclusions drawn from the results obtained.

\section{Data and Study Area}

This section provides brief information on the data and study area considered for the research.

\subsection{Land Satellite 8 (Landsat 8 ) Data}

Land Satellite (Landsat) 8 data was accessed by U.S. Geological Survey (USGS) website [17]. It originally consisted of eleven bands; Coastal Aerosol $(0.43-0.45 \mu \mathrm{m})$, Blue $(0.45-0.51 \mu \mathrm{m})$, Green $(0.53-0.59 \mu \mathrm{m})$, Red $(0.64-0.67 \mu \mathrm{m})$, Near Infrared (NIR) $(0.85-0.88 \mu \mathrm{m})$, Short-Wave Infrared-1 (SWIR 1) $(1.57-1.65 \mu \mathrm{m})$, Short-Wave Infrared-2 (SWIR 2) (2.11- $2.29 \mu \mathrm{m})$, Panchromatic $(0.50-0.68 \mu \mathrm{m})$, Cirrus (1.36-1.38 $\mu \mathrm{m})$, Thermal Infrared (TIRS ) $1(10.60-11.19 \mu \mathrm{m})$, and Thermal Infrared (TIRS ) 2 (11.50-12.51 $\mu \mathrm{m})$ [14]. The first eight bands were made use of in this study. The Spatial resolution of the first seven bands is $30 \mathrm{~m}$, Panchromatic is $15 \mathrm{~m}$, Cirrus is $30 \mathrm{~m}$, and TIRS 1 and TIRS 2 is $100 \mathrm{~m}$. This data was acquired on 18th of May 2016, which is the summer season and is free from clouds. Layer stacking and resolution merge techniques were employed for preprocessing the data before further exploration.

\subsection{Study Area}

Figure 2 indicates the study area considered and it envelops the North Canara District in Karnataka state, India. It is enclosed by the state of Goa and Belgaum Districts in the North, Haveri and

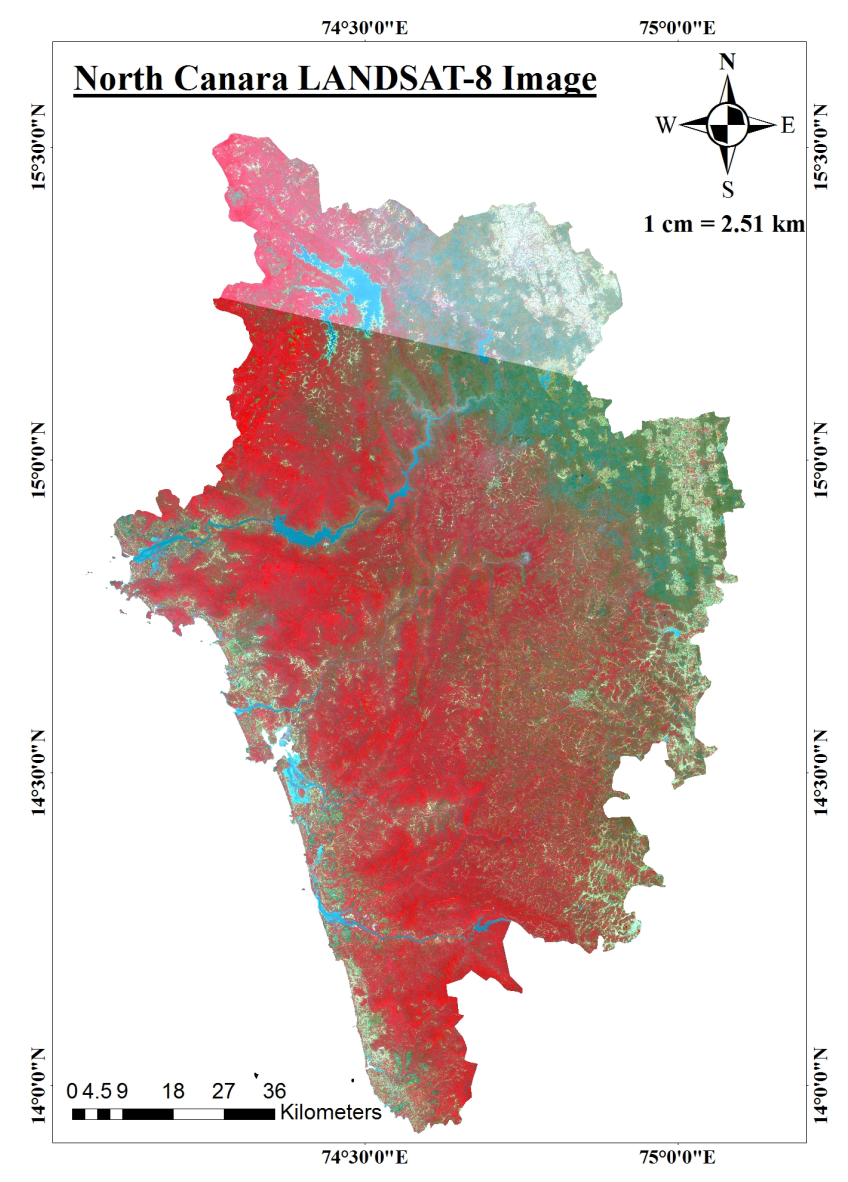

Figure 2: $15 \mathrm{~m}$ spatial resolution map of the study area [17].

Dharwad Districts to the East, Shivamogga and Udupi Districts to the South, and the Arabian Sea to the West. The Western Ghats form the main geographic feature of the region and runs from North to South. To the East of the Western Ghats is the Balaghat upland which is a part of the Deccan Plateau. The typical precipitation on the coastal part is $3000 \mathrm{~mm}$ (120 in) and is as high as $5000 \mathrm{~mm}$ (200 in) in the west-facing slopes of Western Ghats. East facing ridge of the Western Ghats is the rain shadow region and receives, on an average, only $1000 \mathrm{~mm}$ (39 in) rainfall annually [15]. Monsoon receives much of the rainfall, during June-September period. High precipitation within the region supports lavish forests, that coat over $70 \%$ of the coverage area. The north part of the Western Ghats forms Moist Deciduous Forests ranging from 250 to $1000 \mathrm{~m}$ in elevation. Above $1000 \mathrm{~m}$ elevation are the Evergreen rain forests [16].

The study area also has chunks of degraded scrub jungles and savannah. The beach region is characterized by coconut plantations and screw pine. The study area is recognized as a coastal agro-climatic zone by the government of India. The overall spatial resolution of the image is $15 \mathrm{~m}$.

\section{Feature Extraction and Signature Collection}

There are many strategies for collecting the training data, including; in-situ data collection, on-screen selection of polygonal data, and/or on-screen seeding of training data. Each pixel $X$ in the training data is associated with a specific class $c$, is then represented by a 


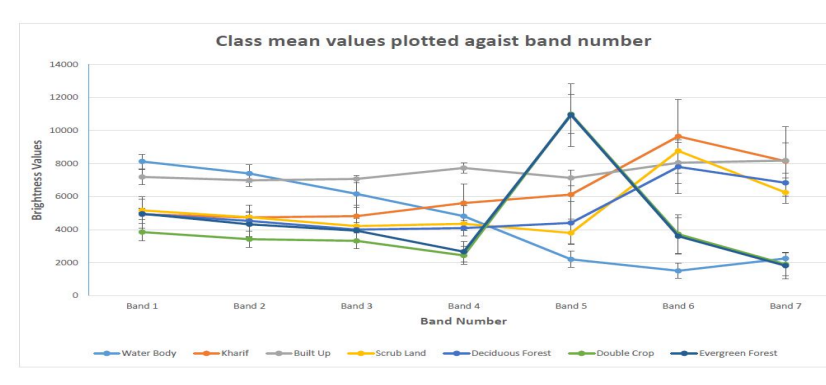

Figure 3: Mean values of classes plotted against band number with standard deviation values as error bars, for classical MLC.

Table 1: Class separability in terms of spectral similarity index for classical MLC.

\begin{tabular}{llllll}
\hline $\begin{array}{l}\text { Class } \\
\text { Pairs }\end{array}$ & $E D^{a}$ & $\begin{array}{l}\text { Class } \\
\text { Pairs }\end{array}$ & $E D^{a}$ & $\begin{array}{l}\text { Class } \\
\text { Pairs }\end{array}$ & $E D^{a}$ \\
\hline $1: 2$ & 1 & $2: 4$ & 0.901 & $3: 7$ & 0.808 \\
$1: 3$ & 0.9 & $2: 5$ & 0.804 & $4: 5$ & 0.282 \\
$1: 4$ & 0.409 & $2: 6$ & 0.134 & $4: 6$ & 0.886 \\
$1: 5$ & 0.556 & $2: 7$ & 0.865 & $4: 7$ & 0.291 \\
$1: 6$ & 0.938 & $3: 4$ & 0.989 & $5: 6$ & 0.794 \\
$1: 7$ & 0.563 & $3: 5$ & 0.80 & $5: 7$ & 0.116 \\
$2: 3$ & 0.967 & $3: 6$ & 0.116 & $6: 7$ & 0.85 \\
\hline \hline
\end{tabular}

1: Built Up, 2: Double Crop/Horticultural Plantations, 3: Water Body, 4: Kharif, 5: Moist Deciduous Forest, 6: Evergreen Forest, 7: Scrub Land.

measurement vector, $X_{c}$, given by [1];

$$
X_{c}=\left[\begin{array}{c}
B V_{i, j, 1} \\
B V_{i, j, 2} \\
B V_{i, j, 3} \\
\vdots \\
B V_{i, j, k}
\end{array}\right]
$$

where, $B V_{i, j, k}$ is the brightness value for the $i, j^{t h}$ pixel in band $k$. The measurement vector also can be used to create a covariance matrix for every class $c$ as [1];

$V_{c}=V_{c k l}=\left[\begin{array}{cccc}\operatorname{cov}_{c 11} & \operatorname{cov}_{c 12} & \ldots & \operatorname{cov}_{c 1 n} \\ \operatorname{cov}_{c 21} & \operatorname{cov}_{c 22} & \ldots & \operatorname{cov}_{c 2 n} \\ : & : & : & : \\ \operatorname{cov}_{c n 1} & \operatorname{cov}_{c n 2} & \ldots & \operatorname{cov}_{c n m}\end{array}\right]$

where, $\operatorname{cov}_{c k l}$ is the covariance of class $c$ between bands $k$ through $l$. By employing an on-screen selection of polygonal data technique, seven land use land cover classes were identified over the study area; i) Water Body, ii) Kharif, iii) Built Up, iv) Scrub Land, v) Double Crop/Horticultural Plantations, vi) Moist Deciduous Forest, and vii) Evergreen Forest. Spectral signatures were collected for each class with a minimum of 300 sample pixels per class.

Two sets of signature were collected for conducting the study. In the first set, only pure pixels were collected from the study area. This signature set is employed for classifying the study area using classical MLC. The second set of signature consists of both pure and mixed pixels. To handle mixed pixel issue, Fuzzy theory based MLC was implemented that uses the second signature set for classification. Class separability was measured in terms of Euclidean distance considering two classes at a time. The considered study area exhibits characteristics of a heterogeneous dataset with more than two class pairs showing severe spectral overlapping. The Euclidean distance was normalized to measure the severity of land cover overlapping. Highest class separability was measured between a class pair was considered as the reference for other classes. All other class pair

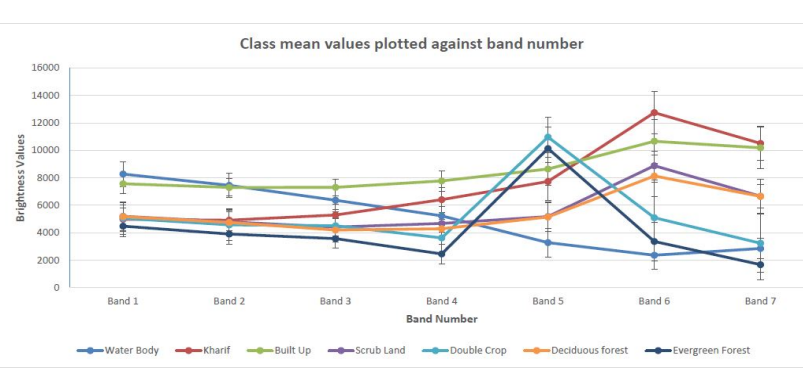

Figure 4: Mean values of classes plotted against band number with standard deviation values as error bars, for Fuzzy theory based MLC.

Table 2: Class separability in terms of spectral similarity index for Fuzzy based MLC.

\begin{tabular}{llllll}
\hline $\begin{array}{l}\text { Class } \\
\text { Pairs }\end{array}$ & $E D^{a}$ & $\begin{array}{l}\text { Class } \\
\text { Pairs }\end{array}$ & $E D^{a}$ & $\begin{array}{l}\text { Class } \\
\text { Pairs }\end{array}$ & $E D^{a}$ \\
\hline $1: 2$ & 1 & $2: 4$ & 0.441 & $3: 7$ & 0.963 \\
$1: 3$ & 0.879 & $2: 5$ & 0.796 & $4: 5$ & 0.544 \\
$1: 4$ & 0.628 & $2: 6$ & 0.486 & $4: 6$ & 0.061 \\
$1: 5$ & 0.666 & $2: 7$ & 0.968 & $4: 7$ & 0.649 \\
$1: 6$ & 0.598 & $3: 4$ & 0.528 & $5: 6$ & 0.518 \\
$1: 7$ & 0.668 & $3: 5$ & 0.774 & $5: 7$ & 0.210 \\
$2: 3$ & 0.338 & $3: 6$ & 0.562 & $6: 7$ & 0.612 \\
\hline \hline \multicolumn{5}{c}{$E D^{a}$ is Normalized Euclidean Distance. }
\end{tabular}

1: Water Body, 2: Kharif, 3: Built Up, 4: Scrub Land, 5: Double Crop/Horticultural Plantations, 6: Moist Deciduous Forest, 7: Evergreen Forest.

separabilities were divided by the reference class separability to produce spectral similarity index (SSI) for class pair in that signature set.

Class pairs with a spectral similarity index value of less than 0.4 were observed to be severely overlapping each other at bound locations. This can be seen by the graph of pixel values plotted against band number in Figure 3 and Figure 4 for classical MLC and Fuzzy theory based MLC signature set, respectively. Spectral similarity index measurements for classical MLC and Fuzzy theory based MLC are shown in Table 1 and Table 2, respectively. It should be noted that severely overlapping classes in the classical MLC now produce relaxed overlapping by the selection of mixed pixels. However, it is still not above the threshold of 0.4 .

\section{Classification Techniques}

This section presents the two classification techniques employed in the study.

\subsection{Classical Maximum Likelihood Classifier}

The Maximum Likelihood Classifier (MLC) assumes that while training the algorithm, the training data statistics for every class in each band are Gaussian distributed. It assigns a pixel to a class $i$ if the calculated probability function for class $i$ is maximum. For the purpose of demonstration, let us consider a universal set $X$ such that its elements are defined by $x$, i.e., $X=\{x\}$. Membership functions for a traditional or classical set $A$ of $X$ can be treated as a binary function $X_{A}$ from $X(0$ or 1$)$ such that $X_{A}=1$ if and only if $x \in A$ otherwise $X_{A}=0$. No intermediate values of the range $[0,1]$ can be viewed in classical set theory. The estimated probability density function for class $w_{i}$ is calculated as [1]:

$\hat{p}\left(x / w_{i}\right)=\frac{1}{(2 \pi)^{\frac{1}{2}} \hat{\sigma}_{i}} \exp \left[-\frac{1}{2} \frac{\left(x-\hat{\mu}_{i}\right)^{2}}{\hat{\sigma}_{i}^{2}}\right]$

where, $x$ is one of the brightness values on the $\mathrm{x}$-axis, $\hat{\mu}_{i}$ is the 
estimated mean of class $w_{i}$, and $\hat{\sigma}_{i}^{2}$ is the estimated variance of class $w_{i}$. Thus, MLC needs to store only mean and variance values of each training class for computing $\hat{p}\left(x / w_{i}\right)$ [1]. For multi-band RS data, 3 can be modified to compute $n$-dimensional multivariate normal density function as [18];

$p\left(X / w_{i}\right)=\frac{1}{(2 \pi)^{\frac{n}{2}}\left|V_{i}\right|^{\frac{1}{2}}} \exp \left[-\frac{1}{2}\left(X-M_{i}\right)^{T} V_{i}^{-1}\left(X-M_{i}\right)\right]$

where, $\left|V_{i}\right|$ is the determinant of the covariance matrix, $V_{i}^{-1}$ is the inverse of the covariance matrix, and $\left(X-M_{i}\right)^{T}$ is the transpose of the vector $\left(X-M_{i}\right)$. The mean vectors $M_{i}$ and covariance matrix $V_{i}$ for each class are estimated from the training data [1]. As Swain and Davis quote, "if we assume there are $m$ classes, then $p\left(X / w_{i}\right)$ is the probability density function for the measurement vector $X$, given that $X$ is from a pattern in class $w_{i}$ " [18]. The classical maximum likelihood decision rule can then be given as;

Decide $X \in w_{i}$ if, and only if,

$p\left(X / w_{i}\right) p\left(w_{i}\right) \geq p\left(X / w_{j}\right) p\left(w_{j}\right)$

for all $i$ and $j$ out of $1,2, \ldots, m$ classes.

That is, the classical MLC assigns a pixel in an RS multi-band image with a measurement vector $X$ to a class for which the product $p\left(X / w_{i}\right) p\left(w_{i}\right)$ is maximum [18]. However, when prior class probabilities are not known, MLC assigns a pixel with measurement vector $X$ to class $i$ if, and only if [1], [18], [19]

$p_{i} \geq p_{j}$

for all $i$ and $j$ out of $1,2, \ldots, m$ classes.

where, $p_{i}$ is given by [1], [18];

$p_{i} \geq-\frac{1}{2} \log _{e}\left|V_{i}\right|-\left[\frac{1}{2}\left(X-M_{i}\right)^{T} V_{i}^{-1}\left(X-M_{i}\right)\right]$

\subsection{Fuzzy Topology Based Maximum Likelihood Classi- fier}

Zadeh's concept of Fuzzy set theory has provided some very useful options for working with heterogeneous datasets [20]. Hard classifiers such as classical MLC hinders the performance of classification in the presence of mixed pixels [1]. On the contrast, using Fuzzy set concepts a Fuzzy set $B$ in $X$ can be described with a membership function $f_{B}$ that links each $x$ a real number from 0 to 1 . The more similar the value of $f_{B}$ to 1 , the more chances of $x$ belonging to $B$ [1], [13]. This principle is believed to provide a satisfactory solution for mixed pixel problem.

The Fuzzy topology based ML classifier discussed in this paper works in three stages; i) Fuzzification of the data, ii) Classification, and iii) Defuzzification.

\subsubsection{Fuzzification}

In the Fuzzification step, each pixel on the input image is converted into a pixel measurement vector, $x$, of membership grades. The Fuzzy membership function for any $x$ must lie in the range 0 to 1 , they should all add up to unity, and should be positive values [1], [13]. These characteristics are listed in (8), (9), and (10).

$0 \leq f_{F_{i}}(x) \leq 1$

$\sum_{x \in X} f_{F_{i}}(x)>0$

$\sum_{i=1}^{m} f_{F_{i}}(x)=1$ where, $F_{i}$ is one of the spectral classes, $X$ represents all pixels in the dataset, $x$ is a pixel measurement vector, $m$ is the number of classes, and $f_{F_{i}}$ is the membership function of the Fuzzy set $F_{i}(1 \leq i \leq m)$. All the membership function values are recorded as a Fuzzy partition matrix [1], [13];

$\left[\begin{array}{cccc}f_{F_{1}}\left(x_{1}\right) & f_{F_{1}}\left(x_{2}\right) & \ldots & f_{F_{1}}\left(x_{n}\right) \\ f_{F_{2}}\left(x_{1}\right) & f_{F_{2}}\left(x_{2}\right) & \ldots & f_{F_{2}}\left(x_{n}\right) \\ : & : & : & : \\ f_{F_{m}}\left(x_{1}\right) & f_{F_{m}}\left(x_{2}\right) & \ldots & f_{F_{m}}\left(x_{n}\right)\end{array}\right]$

where, $n$ represents the total number of pixels, and $x_{i}$ is the $i^{t h}$ pixel's measurement vector.

\subsubsection{Classification}

Fuzzy based classification involves the use of Fuzzy mean and covariance matrices. For class $c$, Fuzzy mean is computed as [13],

$\mu_{c}^{\star}=\frac{\sum_{i=1}^{n} f_{c}\left(x_{i}\right) x_{i}}{\sum_{i=1}^{n} f_{c}\left(x_{i}\right)}$

where, $x_{i}$ is the sample pixel measurement vector $(1 \leq i \leq n), f_{c}$ is the membership function of class $c$, and $n$ is the total number of sample pixel measurement vectors.

The Fuzzy covariance matrix $V_{c}^{\star}$ is computed as [13];

$V_{c}^{\star}=\frac{\sum_{i=1}^{n} f_{c}\left(x_{i}\right)\left(x_{i}-\mu_{c}^{\star}\right)\left(x_{i}-\mu_{c}^{\star}\right)^{T}}{\sum_{i=1}^{n} f_{c}\left(x_{i}\right)}$

These mean and covariance values, as in (12) and (13), replace the conventional mean and covariance matrix in the classical MLC algorithm. This will convert a classical MLC algorithm into a Fuzzy based soft classification algorithm [1].

Fuzzy set theory only provides membership functions for each pixel over the defined number of classes and requires a parametric rule for assigning those pixels to relevant classes. Parametric rules such as Maximum Likelihood, Minimum Distance to Mean and others can be used in the process. This study employs Maximum Likelihood classifier as the parametric rule.

The likelihood function for a pixel belonging to class $k$ in Fuzzy based MLC is given by [1], [13],

$p^{\star}\left(x / w_{i}\right)=\frac{1}{(2 \pi)^{\frac{n}{2}}\left|V_{k}^{\star}\right|^{\frac{1}{2}}} \exp \left[-\frac{1}{2}\left(x-\mu_{k}^{\star}\right)^{T} V_{k}^{\star-1}\left(x-\mu_{k}^{\star}\right)\right]$

Similarly, $p^{\star}\left(x / w_{i}\right)$ is calculated for each pixel for all classes. A membership function then enables the algorithm to decide to which class the corresponding pixel is to be assigned. For Maximum Likelihood classifier, the membership function can be defined as [13];

$f_{c}(x)=\frac{p^{\star}\left(x / w_{k}\right)}{\sum_{i=1}^{m} p^{\star}\left(x / w_{k}\right)}$

The membership grades of a pixel vector depend on $x$ 's position in the vector space. $f_{c}(x)$ increases exponentially with the decrease of $\left(x-\mu_{k}^{\star}\right)^{T} V_{k}^{\star-1}\left(x-\mu_{k}^{\star}\right)$, that is, the Mahalanobis distance between pixel $x$ and class $k$. The factor $\sum_{i=1}^{m} p^{\star}\left(x / w_{k}\right)$ is a normalization factor

[13]. This logic creates a membership grade matrix for each pixel.

The Fuzzy topology based ML classification in ERDAS Imagine v2014 ${ }^{\circledR}$ creates n-layer classification and distance files. Using the Fuzzy convolution utility, a moving window convolution is performed on the fuzzy classification with multiple output class assignments [21]. 


\subsubsection{Defuzzification using Fuzzy Convolution}

Fuzzy convolution method is employed to convert the $n$ layer output of classification into a map like structure. It creates the map by computing the total weighted inverse distance of all the classes in a window of pixels. The process first computes the total inverse distance summed over the entire set of Fuzzy classification layers for each class. It then assigns the center pixel to the class for which this total $T[k]$, is largest. The total inverse distance can be computed using [21]:

$$
T[k]=\sum_{i=0}^{s} \sum_{j=0}^{s} \sum_{l=0}^{n} \frac{w_{i j}}{D_{i j l}[k]}
$$

where, $i$ is the row index of window, $j$ is the column index of window, $s$ is the size of window, $l$ is the layer index of fuzzy layers used, $n$ is the number of fuzzy layers used, $W$ is the weight table for window, $k$ is the class value, $D[k]$ is the distance file value for class $k$, and $T[k]$ is the total weighted distance of window for class $k$ [21]. This study considers a $5 \times 5$ size deconvolution window given by;

$W=\left[\begin{array}{ccccc}0.50 & 0.605 & 0.646 & 0.605 & 0.5 \\ 0.605 & 0.75 & 0.823 & 0.75 & 0.605 \\ 0.646 & 0.823 & 1 & 0.0 .823 & 0.646 \\ 0.605 & 0.75 & 0.823 & 0.75 & 0.605 \\ 0.50 & 0.605 & 0.646 & 0.605 & 0.5\end{array}\right]$

\section{Accuracy Assessment}

Accuracy assessment is the most ordinarily used technique for the analysis of classification results. The accuracy assessment analysis is mainly presented by the use of error matrix [2], [22]. Accuracy assessment begins by selecting a certain number of ground reference points or pixels on the classified map that is allotted to a class by the classifier algorithm, and verify their correctness. This study aims at obtaining a minimum of $85 \%$ classification accuracy. To satisfy this constraint, the minimum number of pixels required for accuracy assessment is given by [2],

$N=\frac{4 P(1-P)}{e^{2}}$

where, $N$ is the minimum number of pixels required for accuracy assessment, $P$ is the map accuracy expected (in \%), and $e$ is the error margin acceptable. For $85 \%$ of expected classification accuracy, $N=319$. However, this study considers 1000 ground reference points for the process of accuracy assessment.

The following accuracy assessment metrics have been computed from the confusion matrix for analysing the results obtained during the study.

$$
\text { Produccer's Accuracy }(P A)=\frac{n_{i i}}{\sum_{i=1}^{M} n_{i k}} \times 100
$$

User's Accuracy $(U A)=\frac{n_{i i}}{\sum_{i=1}^{M} n_{k i}} \times 100$

Omission Error $(O E)=\left(1-\frac{n_{i i}}{\sum_{i=1}^{M} n_{i k}}\right) \times 100$

Comission Error $(C E)=\left(1-\frac{n_{i i}}{\sum_{i=1}^{M} n_{k i}}\right) \times 100$
Table 3: Suggested ranges for the Kappa Coefficient [2].

\begin{tabular}{cc}
\hline \hline Kappa Coefficient & Classification can be regarded as \\
\hline Below 0.4 & Poor \\
$0.41-0.60$ & Moderate \\
$0.61-0.75$ & Good \\
$0.76-0.80$ & Excellent \\
0.81 and above & Almost Perfect \\
\hline \hline
\end{tabular}

Overall Classification Accuracy $(O C A)=\frac{\sum_{i=1}^{M} n_{i i}}{N}$

$k_{\text {hat }}=\frac{\text { prob. of correct classification }- \text { prob. of chance agreement }}{1-\text { prob. of chance agreement }}$

$k_{\text {hat }}=\frac{N \sum_{i=1}^{M} n_{i i}-\sum_{i=1}^{M} n_{+i} n_{i+}}{N^{2}-\sum_{i=1}^{M} n_{+i} n_{i+}}$

$k_{\text {hat_class }}=\frac{N n_{i i}-n_{+i} n_{i+}}{N n_{+i}-n_{+i} n_{i+}}$

Quantity Disagreement $(Q)=\frac{1}{2} \sum_{i=1}^{M}\left|p_{+i}-p_{i+}\right|$

Allocation Disagreement $(A)=\sum_{i=1}^{M} \min \left\{\left(p_{+i}-p_{i i}\right),\left(p_{i+}-p_{i i}\right)\right\}$

where, $n_{i i}$ is the diagonal element of class $i, \sum_{i=1}^{M} n_{i k}$ is the column total of class $i$ and $\sum_{i=1}^{M} n_{k i}$ is the row total of class $i$, and $M$ is the total number of classes, $p_{+i}$ is the proportion of row total for class $i$ and $p_{i+}$ is the proportion of the column total for class $i,\left(p_{+i}-p_{i i}\right)$ represents the Commission Error (CE) and $\left(p_{i+}-p_{i i}\right)$ represents the Omission Error (OE). Table 3 presents the relation between a range of Kappa coefficients and the correctness of classified map [2].

Lastly, the total error which is the disagreement between the reference data and the classified map can be viewed as the sum of Quantity disagreement and Allocation disagreement, given by [2],

$1-P=A+Q($ in $\%)$

\section{Results and Discussions}

In this section, results obtained during the study are presented and discussed. In the first subsection, results obtained for classical maximum likelihood classifier are presented. In the second subsection, results obtained for Fuzzy topology based maximum likelihood classifier are presented. 
Table 4: Results of classical maximum likelihood classification.

\begin{tabular}{|c|c|c|c|c|c|c|c|c|}
\hline Class Name & $\begin{array}{l}\text { Reference } \\
\text { Totals }\end{array}$ & $\begin{array}{l}\text { Classified } \\
\text { totals }\end{array}$ & $\begin{array}{l}\text { Number } \\
\text { Correct }\end{array}$ & $\begin{array}{l}\text { Producer's } \\
\text { Accuracy } \\
(\%)\end{array}$ & $\begin{array}{l}\text { Omission } \\
\text { Error } \\
(\%)\end{array}$ & $\begin{array}{l}\text { User's } \\
\text { Accuracy } \\
(\%)\end{array}$ & $\begin{array}{l}\text { Commission } \\
\text { Error }(\%)\end{array}$ & $\begin{array}{l}\text { Kappa } \\
\text { Value } \\
\left(k_{\text {hat }}\right)\end{array}$ \\
\hline Evergreen Forest & 79 & 32 & 21 & 26.58 & 73.42 & 65.63 & 34.37 & 0.6268 \\
\hline Scrub Land & 235 & 269 & 167 & 71.06 & 28.94 & 62.08 & 37.92 & 0.5043 \\
\hline Moist Deciduous Forest & 59 & 1 & 1 & 1.69 & 98.31 & 100.00 & 0.00 & 1 \\
\hline Built Up & 5 & 24 & 1 & 20.00 & 80.00 & 4.17 & 95.83 & 0.0369 \\
\hline Double Crop/ Horticultural Plantations & 534 & 358 & 353 & 66.10 & 33.90 & 98.60 & 1.40 & 0.97 \\
\hline Water Body & 57 & 313 & 49 & 85.96 & 14.04 & 15.65 & 84.35 & 0.1056 \\
\hline Kharif & 31 & 3 & 3 & 9.68 & 90.32 & 100.00 & 0.00 & 1 \\
\hline Row Total & 1000 & 1000 & 595 & & & & & \\
\hline Overall Classification Accuracy & $59.50 \%$ & & & & & & & \\
\hline Overall Kappa Statistic & 0.4414 & & & & & & & \\
\hline Quantity Disagreement (Q) & $0.5 \times(618$ & $8 / 1000) \times 1$ & $100=30.9$ & $\%$ & & & & \\
\hline Allocation Disagreement (A) & $(96 / 1000)$ & $\times 100=09$ & $9.60 \%$ & & & & & \\
\hline Total Error $\rightarrow\left(1-p_{0}\right)=\mathrm{A}+\mathrm{Q}$ & $\begin{array}{l}(100-59 \\
40.5 \%=\end{array}$ & $\begin{array}{l}50) \%=((3) \\
40.5 \%\end{array}$ & $309+96) /$ & $000) \times 100$ & & & & \\
\hline
\end{tabular}

\subsection{Results of Classical Maximum Likelihood Classifica- tion}

Figure 5 shows the classical maximum likelihood classified map of the study area and Table 4 indicates the results obtained through the analysis of error matrix for the same. 10 pixels were selected randomly from the study area for analysing the pixel assignment process. These pixels are listed in Table 5 with the corresponding row number, column number, and its LULC class on the input image. From Table 6 it is determined that classical ML classifier fails to

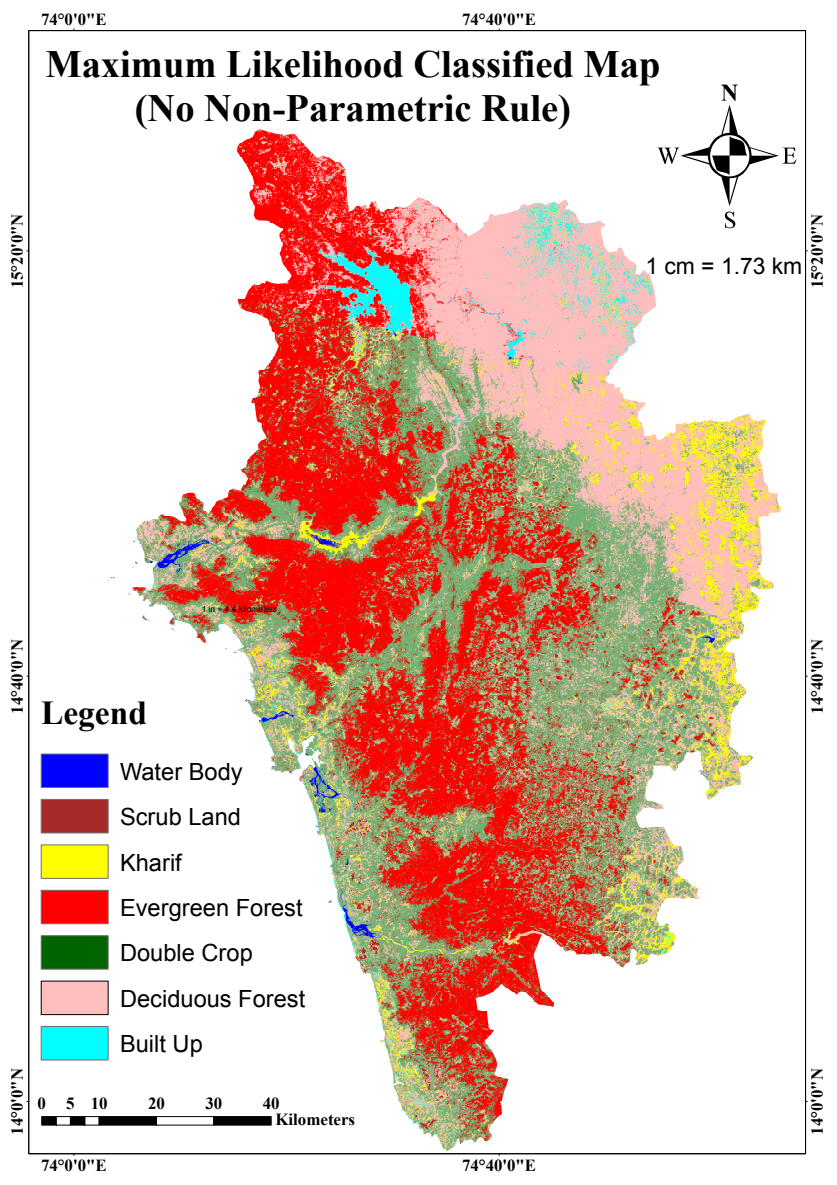

Figure 5: Classical maximum likelihood classified map of the study area. proficiently classify all of the selected pixels into their corresponding LULC class. Pixel 1, 8, 9, and 10 were classified correctly by conventional MLC whereas other pixels were misclassified. This is believed to be the effect of spectral overlapping on the performance of ML classifier.

Classical MLC extracted Water Body and Scrub Land classes with highest possible User's Accuracy (UA) of 100\%. Evergreen Forest class was also excellently extracted with a User's Accuracy of 98.60\%. Kharif and Moist Deciduous Forest classes were moderately extracted. Double Crop/Horticultural Plantations and Built Up classes were very poorly extracted by ML classifier.

The reduction in class accuracy produced by classical MLC is attributed to spectral overlapping of Double Crop/Horticultural Plantations with Evergreen Forest and Built Up with Kharif and Water Body classes. The overall classification accuracy was recorded as $59.50 \%$ with an overall kappa value of 0.4414 . With reference to Table 2, the overall classification performance of the classical MLC over the considered study area can be termed as moderate.

Result analysis is performed by calculating three parameters; Mahalanobis distance between input pixel feature vector, $X$, and mean of each class $M_{i}$, i.e., $\left[\left(X-M_{i}\right) V_{i}^{-1}\left(X-M_{i}\right)^{T}\right], p_{i}$ as in (7), and multivariate Gaussian density function $p\left(X / w_{i}\right)$ as in (4). The calculated values of these parameters are listed in Table 6 .

Table 5: Pixels considered for analysing the assignment strategy.

\begin{tabular}{|c|c|c|c|}
\hline $\begin{array}{l}\text { Pixel } \\
\text { no. }\end{array}$ & Row & Column & Ground Truth Class \\
\hline 1 & 6500 & 4020 & Evergreen Forest \\
\hline 2 & 6840 & 4608 & Scrub Land \\
\hline 3 & 8177 & 6313 & Moist Deciduous Forest \\
\hline 4 & 5539 & 7118 & Built Up \\
\hline 5 & 5544 & 7117 & $\begin{array}{l}\text { Double Crop/ Horticultural Plan- } \\
\text { tations }\end{array}$ \\
\hline 6 & 7050 & 2284 & Water Body \\
\hline 7 & 3452 & 5760 & Kharif \\
\hline 8 & 3952 & 6897 & Kharif \\
\hline 9 & 3846 & 6904 & Built Up \\
\hline 10 & 3890 & 7016 & $\begin{array}{l}\text { Double Crop/ Horticultural Plan- } \\
\text { tations }\end{array}$ \\
\hline
\end{tabular}


Table 6: Class assignment metrics for classical maximum likelihood classification.

\begin{tabular}{|c|c|c|c|c|c|c|c|c|c|}
\hline Pixel No. & Metric & $E G F^{a}$ & $S L^{b}$ & $M D F^{c}$ & $B U^{d}$ & $D C / H P^{e}$ & $W B^{f}$ & $K H^{g}$ & Class Assigned \\
\hline \multirow{3}{*}{ pixel 1} & $M D^{a}$ & 5.582 & 3557.910 & 1082.858 & 3398.986 & 27.405 & 88073.294 & 3156.180 & \multirow{3}{*}{$E G F^{a}$} \\
\hline & $\left(\hat{p}_{i}\right)$ & -37.607 & -1810.524 & -578.395 & -1735.266 & -50.501 & -44063.117 & -1613.559 & \\
\hline & $\hat{p}\left(X / w_{i}\right)$ & -44.038 & -1816.955 & -584.826 & -1741.696 & -56.932 & -44069.547 & -1619.990 & \\
\hline \multirow{3}{*}{ pixel 2} & $M D^{a}$ & 29.988 & 2850.020 & 684.584 & 2699.843 & 22.628 & 69694.401 & 2354.420 & \multirow{3}{*}{$D C / H P^{e}$} \\
\hline & $\left(\hat{p}_{i}\right)$ & -49.810 & -1456.579 & -379.258 & -1385.694 & -48.113 & -34873.670 & -1212.679 & \\
\hline & $\hat{p}\left(X / w_{i}\right)$ & -56.241 & -1463.010 & -385.689 & -1392.125 & -54.543 & -34880.101 & -1219.110 & \\
\hline \multirow{3}{*}{ pixel 3} & $M D^{a}$ & 224.732 & 584.352 & 82.616 & 1428.182 & 69.893 & 100289.838 & 283.520 & \multirow{3}{*}{$D C / H P^{e}$} \\
\hline & $\left(\hat{p}_{i}\right)$ & -147.182 & -323.745 & -78.274 & -749.864 & -71.745 & -50171.389 & -177.229 & \\
\hline & $\hat{p}\left(X / w_{i}\right)$ & -153.613 & -330.176 & -84.705 & -756.294 & -78.176 & -50177.819 & -183.660 & \\
\hline \multirow{3}{*}{ pixel 4} & $M D^{a}$ & 402.487 & 393.564 & 20.098 & 1041.250 & 138.511 & 128059.796 & 21.285 & \multirow{3}{*}{$K H^{g}$} \\
\hline & $\left(\hat{p}_{i}\right)$ & -236.059 & -228.351 & -47.015 & -556.398 & -106.054 & -64056.368 & -46.112 & \\
\hline & $\hat{p}\left(X / w_{i}\right)$ & -242.490 & -234.782 & -53.446 & -562.828 & -112.485 & -64062.799 & -52.543 & \\
\hline \multirow{3}{*}{ pixel 5} & $M D^{a}$ & 428.484 & 561.440 & 35.523 & 1074.357 & 162.477 & 134941.281 & 28.858 & \multirow{3}{*}{$K H^{g}$} \\
\hline & $\left(\hat{p}_{i}\right)$ & -249.058 & -312.289 & -54.727 & -572.951 & -118.037 & -67497.110 & -49.898 & \\
\hline & $\hat{p}\left(X / w_{i}\right)$ & -255.489 & -318.720 & -61.158 & -579.382 & -124.468 & -67503.541 & -56.329 & \\
\hline \multirow{3}{*}{ pixel 6} & $M D^{a}$ & 2593.934 & 255.099 & 357.506 & 405.393 & 771.550 & 132094.992 & 113.008 & \multirow{3}{*}{$K H^{g}$} \\
\hline & $\left(\hat{p}_{i}\right)$ & -1331.783 & -159.119 & -215.719 & -238.469 & -422.574 & -66073.966 & -91.973 & \\
\hline & $\hat{p}\left(X / w_{i}\right)$ & -1338.214 & -165.550 & -222.150 & -244.900 & -429.005 & -66080.397 & -98.404 & \\
\hline \multirow{3}{*}{ pixel 7} & $M D^{a}$ & 612.408 & 96.761 & 1.442 & 378.153 & 265.093 & 61119.275 & 55.867 & \multirow{3}{*}{$M D F^{c}$} \\
\hline & $\left(\hat{p}_{i}\right)$ & -341.020 & -79.950 & -37.687 & -224.849 & -169.345 & -30586.107 & -63.402 & \\
\hline & $\hat{p}\left(X / w_{i}\right)$ & -347.451 & -86.380 & -44.118 & -231.280 & -175.776 & -30592.538 & -69.833 & \\
\hline \multirow{3}{*}{ pixel 8} & $M D^{a}$ & 2835.231 & 122.171 & 292.038 & 966.333 & 589.745 & 212619.842 & 18.308 & \multirow{3}{*}{$K H^{g}$} \\
\hline & $\left(\hat{p}_{i}\right)$ & -1452.432 & -92.655 & -182.985 & -518.939 & -331.671 & -106336.391 & -44.623 & \\
\hline & $\hat{p}\left(X / w_{i}\right)$ & -1458.862 & -99.086 & -189.416 & -525.370 & -338.102 & -106342.822 & -51.054 & \\
\hline \multirow{3}{*}{ pixel 9} & $M D^{a}$ & 2933.614 & 2849.388 & 773.271 & 132.324 & 479.855 & 44212.412 & 183.005 & \multirow{3}{*}{$B U^{d}$} \\
\hline & $\left(\hat{p}_{i}\right)$ & -1501.623 & -1456.263 & -423.601 & -101.934 & -276.726 & -22132.676 & -126.972 & \\
\hline & $\hat{p}\left(X / w_{i}\right)$ & -1508.054 & -1462.694 & -430.032 & -108.365 & -283.157 & -22139.106 & -133.402 & \\
\hline \multirow{3}{*}{ pixel 10} & $M D^{a}$ & 162.537 & 5094.447 & 1230.853 & 4529.009 & 2.470 & 130994.862 & 4688.687 & \multirow{3}{*}{$D C / H P^{e}$} \\
\hline & $\left(\hat{p}_{i}\right)$ & -116.085 & -2578.793 & -652.392 & -2300.277 & -38.033 & -65523.901 & -2379.813 & \\
\hline & $\hat{p}\left(X / w_{i}\right)$ & -122.515 & -2585.223 & -658.823 & -2306.708 & -44.464 & -65530.332 & -2386.244 & \\
\hline
\end{tabular}

$E G F^{a}$ : Evergreen Forest, $S L^{b}$ : Scrub Land, $M D F^{c}$ : Moist deciduous Forest, $B U^{d}$ : Built Up, $D C / H P^{e}$ : Double Crop/ Horticultural plantations, $W B^{f}$ : water Body, and $K H^{g}$ : Kharif

Table 7: Results of Fuzzy based maximum likelihood classification.

\begin{tabular}{|c|c|c|c|c|c|c|c|c|}
\hline Class Name & $\begin{array}{l}\text { Reference } \\
\text { Totals }\end{array}$ & $\begin{array}{l}\text { Classified } \\
\text { totals }\end{array}$ & $\begin{array}{l}\text { Number } \\
\text { Correct }\end{array}$ & $\begin{array}{l}\text { Producer's } \\
\text { Accuracy } \\
(\%)\end{array}$ & $\begin{array}{l}\text { Omission } \\
\text { Error } \\
(\%)\end{array}$ & $\begin{array}{l}\text { User's } \\
\text { Accuracy } \\
(\%)\end{array}$ & $\begin{array}{l}\text { Commission } \\
\text { Error }(\%)\end{array}$ & $\begin{array}{l}\text { Kappa } \\
\text { Value } \\
\left(k_{\text {hat }}\right)\end{array}$ \\
\hline Evergreen Forest & 534 & 538 & 502 & 94.01 & 05.99 & 93.31 & 06.69 & 0.8564 \\
\hline Scrub Land & 59 & 70 & 40 & 67.80 & 32.20 & 57.14 & 42.86 & 0.5446 \\
\hline Moist Deciduous Forest & 235 & 214 & 205 & 87.23 & 12.77 & 95.79 & 04.21 & 0.9450 \\
\hline Built Up & 5 & 14 & 3 & 60.00 & 40.00 & 21.43 & 78.57 & 0.2103 \\
\hline Double Crop/ Horticultural Plantations & 57 & 80 & 37 & 64.91 & 35.09 & 46.25 & 53.75 & 0.4300 \\
\hline Water Body & 31 & 16 & 16 & 51.61 & 48.39 & 100.00 & 00.00 & 1.0000 \\
\hline Kharif & 79 & 68 & 59 & 74.68 & 25.32 & 86.76 & 13.24 & 0.8563 \\
\hline Row Total & 1000 & 1000 & 862 & & & & & \\
\hline Overall Classification Accuracy & $86.20 \%$ & & & & & & & \\
\hline Overall Kappa Statistic & 0.787 & & & & & & & \\
\hline Quantity Disagreement (Q) & $0.5 \times(94 /$ & $/ 1000) \times 10$ & $0=04.7 \%$ & & & & & \\
\hline Allocation Disagreement (A) & $(91 / 1000)$ & $\times 100=08$ & $.9 \%$ & & & & & \\
\hline Total Error $\rightarrow\left(1-p_{0}\right)=\mathrm{A}+\mathrm{Q}$ & $\begin{array}{l}(100-86 \\
13.8 \%=\end{array}$ & $\begin{array}{l}20) \%=((4) \\
13.8 \%\end{array}$ & $47+91) / 10$ & $00) \times 100$ & & & & \\
\hline
\end{tabular}

\subsection{Results of Fuzzy Theory based Maximum Likelihood Classification}

Figure 6 illustrates the Fuzzy theory based maximum likelihood classification map of the study area. Fuzzy theory based maximum likelihood classifier has shown significant improvement in classification performance. A classification accuracy improvement of $26.70 \%$ was recorded. Also, the overall Kappa value was recorded at 0.7870 , which from Table 2, indicates an excellent performance from the classifier. However, the class accuracies have been observed to show variations in their values as compared to classical MLC. Only Water Body class was observed to retain the same user's accuracy value. Scrub Land and Water Body classes have suffered a small decrease in their user's accuracy values. However, Moist Deciduous Forest, Built Up, Double Crop/Horticultural Plantations and Kharif classes have seen an increase in user's accuracy values resulting in a superior 
Table 8: Total inverse weighted distance of all classes from the weight windows for selected pixels.

\begin{tabular}{llllllllllll}
\hline \hline $\begin{array}{l}\text { Pixel } \\
\text { No. }\end{array}$ & Row & Column & $\begin{array}{l}\text { LULC } \\
\text { Class }\end{array}$ & $E G F^{a}$ & $S L^{b}$ & $M D F^{c}$ & $B U^{d}$ & $D C / H P^{e}$ & $W B^{f}$ & $K H^{g}$ & $\begin{array}{l}\text { Class } \\
\text { Assigned }\end{array}$ \\
\hline 1 & 6500 & 4020 & $E G F^{a}$ & 0.1988 & 0.1199 & 0.1159 & 0.0654 & 0.1664 & 0.0026 & 0.0463 & $E G F^{a}$ \\
2 & 6840 & 4608 & $S L^{b}$ & 0.1243 & 0.1714 & 0.1593 & 0.0821 & 0.1359 & 0.0011 & 0.1041 & $S L^{b}$ \\
3 & 8177 & 6313 & $M D F^{c}$ & 0.1426 & 0.1592 & 0.1701 & 0.0949 & 0.1348 & 0.0012 & 0.0815 & $M D F^{c}$ \\
4 & 5539 & 7118 & $B U^{d}$ & 0.0639 & 0.1140 & 0.0576 & 0.1655 & 0.1443 & 0.0022 & 0.1345 & $B U^{d}$ \\
5 & 5544 & 7117 & $D C / H P^{e}$ & 0.0635 & 0.1561 & 0.0989 & 0.1361 & 0.1709 & 0.0019 & 0.1431 & $D C / H P^{e}$ \\
6 & 7050 & 2284 & $W B^{f}$ & 0.0468 & 0.0587 & 0.0432 & 0.0951 & 0.0768 & 0.1552 & 0.0701 & $W B^{f}$ \\
7 & 3452 & 5760 & $K H^{g}$ & 0.0148 & 0.1188 & 0.1634 & 0.1366 & 0.0540 & 0.0011 & 0.1748 & $K H^{g}$ \\
8 & 3952 & 6897 & $K H^{g}$ & 0.0295 & 0.1161 & 0.0541 & 0.1059 & 0.0920 & 0.0007 & 0.1939 & $K H^{g}$ \\
9 & 3846 & 6904 & $B U^{d}$ & 0.0215 & 0.0761 & 0.0314 & 0.1742 & 0.0582 & 0.0025 & 0.1235 & $B U^{d}$ \\
10 & 3890 & 7016 & $D C / H P^{e}$ & 0.1791 & 0.0758 & 0.0568 & 0.0326 & 0.2013 & 0.0018 & 0.0229 & $D C / H P^{e}$ \\
\hline \hline
\end{tabular}

$E G F^{a}$ : Evergreen Forest, $S L^{b}$ : Scrub Land, $M D F^{c}$ : Moist Deciduous Forest, $B U^{d}$ : Built Up,

$D C / H P^{e}$ : Deciduous Forest/Horticultural Plantations, $W B^{f}$ : Water Body, $K H^{g}$ : Kharif

overall classification accuracy value compared to that of classical ML classifier. Table 7 indicates the results obtained through the analysis of error matrix for Fuzzy based MLC.

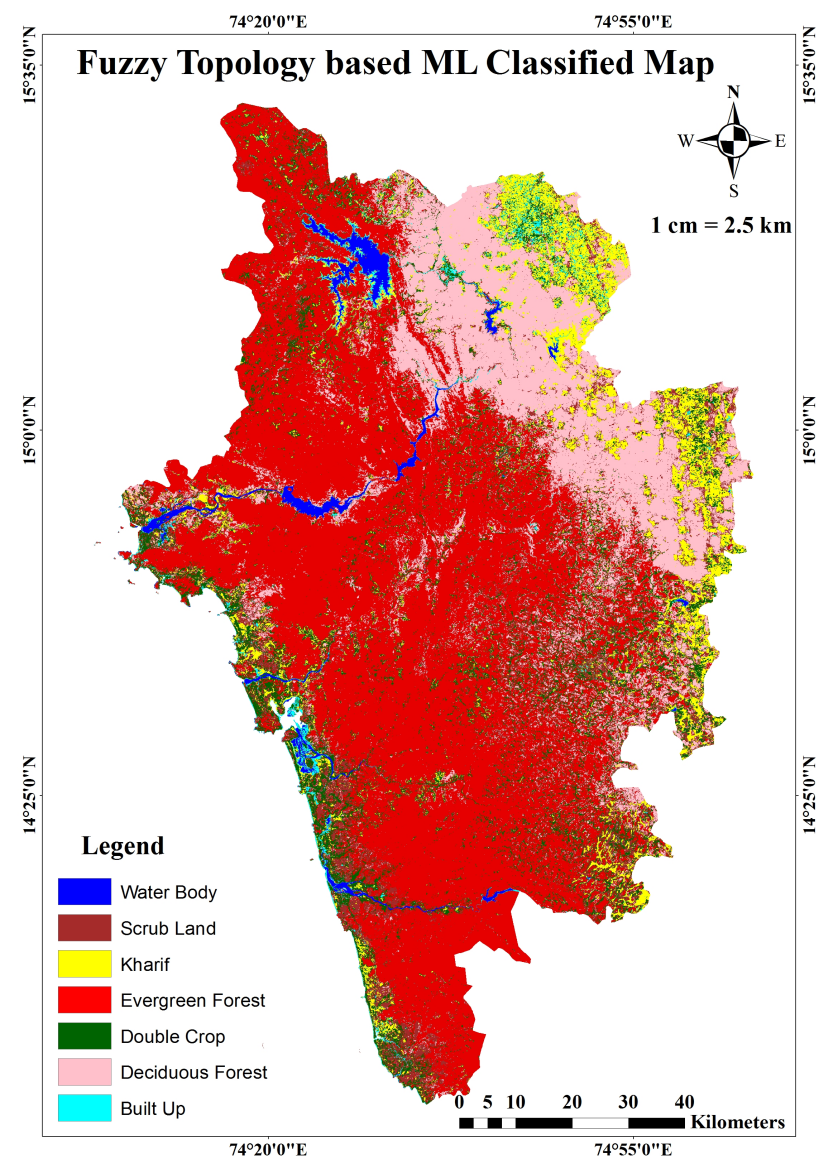

Figure 6: Fuzzy theory based maximum likelihood classified map of the study area.

To illustrate the usefulness of using the inverse weighted distance of all classes from the weight windows for pixels for assigning pixels to class values, Table 8 was constructed for 10 pixels as referred in Table 5. It is observed that all of the considered pixels were correctly classified to their respective classes by using weighted inverse distance as decision metric.

\subsection{Placing Confidence Limits on Assessed Accuracy}

The process of accuracy assessment provides a quantitative analysis of the results of classification. Validation of results obtained through accuracy assessment can be performed by placing a confidence level on the results of accuracy assessment. A straightforward statistical approach may be used to predict the range within which the true value of the classified map accuracy lies, with $95 \%$ certainty. It is possible to use the normal distribution to obtain this interval by the expression [2];

$-Z_{\frac{\alpha}{2}}<\frac{x-n P}{\sqrt{n P(1-P)}}<Z_{\frac{\alpha}{2}}$

where, $n$ is the number of testing pixels, $x(=n p)$ is the number of correctly labelled pixels, $P$ is the thematic map accuracy (in \%) $=p=x / n, Z_{\frac{\alpha}{2}}$ is the value of the normal distribution beyond which on both tails $\alpha$ of the population is excluded [2].

If the normalized statistic $\frac{x-n P}{\sqrt{n P(1-P)}}$ is expected to reside within the $95 \%$ portion of the normal curve, then $Z_{\frac{\alpha}{2}}= \pm 1196(\approx \pm 2)$. From (30), the estimate of the thematic map accuracy, $P$, estimated by the proportion of correctly classified pixels in the testing set, at the $95 \%$ confidence level are [2],

$$
\frac{x \pm 1.921 \pm 1.960 \sqrt{\frac{x(n-x)}{n}+0.960}}{n+3.842}
$$

which, for large values $n$ and $x$, and for reasonable accuracies, is approximated [2],

$\frac{x \pm 1.960 \sqrt{\frac{x(n-x)}{n}+0.960}}{n}=p \pm \frac{1.960}{n} \sqrt{\frac{x(n-x)}{n}+0.960}$

For 1000 testing pixels and a minimum of $80 \%$ of expected accuracy, it is expected to have, at least, 800 pixels to be correctly classified. From 32, the estimated map accuracy is predicted to have bounds within $p=p \pm 0.039$. For 1000 testing pixels, in percentage terms, the map accuracy is predicted to be between $82.1 \%$ and $89.9 \%$. This illustrates that; the classification accuracy obtained by employing Fuzzy theory based ML classifier is in line with the theoretical expectations.

\subsection{Area Coverage Statistics}

According to the statistics obtained from the classified map of the Fuzzy theory based ML classifier, the area covered by each LULC class on the Earth surface is calculated and is as indicated in Table 
Table 9: Area coverage statistics computed using results of Fuzzy based maximum likelihood classification.

\begin{tabular}{llll}
\hline \hline Class Name & Pixel Count & $\begin{array}{l}\text { Area Covered } \\
(\text { Sq. km. })\end{array}$ & $\begin{array}{l}\text { Percentage of } \\
\text { Area Coverage } \\
(\%)\end{array}$ \\
\hline Kharif & 3155364 & 709.9569 & 6.948974 \\
Water Body & 698105 & 157.073625 & 1.537418 \\
Double Crop/Horticultural Plantations & 4023902 & 905.37795 & 8.861732 \\
Built Up & 602860 & 135.6435 & 1.327662 \\
Moist Deciduous Forest & 8733479 & 1965.032775 & 19.23351 \\
Scrub Land & 4031375 & 907.059375 & 8.878189 \\
Evergreen Forest & 24162541 & 5436.571725 & 53.21252 \\
\hline Total Area Covered & & 10216.71585 & 100 \\
\hline Overall Natural Vegetation Coverage & & 8308.663875 & 81.32421 \\
\hline Overall Non-Vegetation Coverage & & 1908.051975 & 18.67579 \\
\hline \hline
\end{tabular}

9. Natural vegetation (Evergreen Forest + Moist Deciduous Forest + Scrub Land) covers $81.324 \%$ and non Natural vegetation (Water Body + Double Crop/Horticultural Plantations + Built Up + Kharif) covers $18.676 \%$ of the total area.

\section{Conclusion}

In this paper, a novel method for improving the classification of large RS imagery through embedding Fuzzy theory into classical Maximum Likelihood Classifier (MLC) is discussed. The Fuzzy theory based MLC separates spectrally overlapping classes with higher precision compared to classical MLC. It produces precise results for large heterogeneous study areas, even with the presence of mixed pixels and spectrally overlapping classes. Fuzzy theory based MLC produced a $26.7 \%$ improvement in classification accuracy compared to classical MLC. Further, the Fuzzy theory based MLC has also shown promising results in improvement of individual class accuracy values indicating its superiority to classical MLC. The objective of obtaining classification results with a confidence level of $95 \%$ with $\pm 4 \%$ error margin is achieved by using Fuzzy theory based MLC. Hence, it can be concluded that the discussed Fuzzy theory based MLC handles mixed pixel issue more successfully. However, it is also observed that embedding Fuzzy theory into classical MLC introduces uncertainties into the classification of some spectral classes which are well extracted by classical MLC. Though this uncertainty is negligible and also the overall classification results are on par with the expected results, further investigation on this could shed more light on such a behaviour.

\section{Acknowledgement}

The authors wish to thank the United States Geological Survey (USGS) for letting us acquire the data for the research.

\section{References}

[1] J.R. Jensen, Introductory digital image processing: a remote sensing perspective, Prentice-Hall Inc., (2000).

[2] J.A. Richards, Remote Sensing Digital Image Analysis- An Introduction, Springer, (2006).

[3] D. Lu, Q. Weng, "A survey of image classification methods and techniques for improving classification performance", International Journal of Remote Sensing, Vol.28, No.5, (2007), pp.823-870.

[4] L. Bruzzone, D.F. Prieto, "Unsupervised Retraining of a MaximumLikelihood Classifier for the Analysis of Multitemporal RemoteSensing Images", Proceedings of the EUROPTO Conference on Image and Signal Processing for Remote Sensing, 3871, (1999), pp.169-174.

[5] D. Peuquet, D. Marble, D. Francis, Introductory Readings in Geographic Information Systems and Remote Sensing, CRC Press, (2003)

[6] Y. Wang, M. Jamshidi, "Fuzzy logic applied in remote sensing image classification", Systems, Man and Cybernetics, Vol.7, (2004), pp.63786382
[7] F. Wang, "Fuzzy Supervised Classification of Remote Sensing Images", IEEE Transactions on Geoscience and Remote Sensing, Vol.28, No.2, (1990), pp.194-201.

[8] J. Zhang, G.M. Foody, "A fuzzy classification of sub-urban land cover from remotely sensed imagery.", International Journal of Remote Sensing, Vol.19, No.14, (1998), pp.2721-2738.

[9] F. Melgani, B.A.R. Al Hashemy, S.M.R. Taha, "An explicit fuzzy supervised classification method for multispectral remote sensing images.", IEEE Transactions on Geoscience and Remote Sensing, Vol.38, No.1, (2000), pp.287-295.

[10] K. Liu, W. Shi, H. Zhang, "A fuzzy theory-based maximum likelihood classification.", ISPRS Journal of Photogrammetry and Remote Sensing, Vol.66, No.1, (2011), pp.103-114

[11] E. Console, M.C. Mouchot, "Fuzzy Classification Techniques in the Urban Area Recognition.", Proceedings of the International Geoscience and Remote Sensing Symposium, (1996), pp.1373-1375.

[12] G. Droj, "The applicability of fuzzy theory in remote sensing image classification", Studia Univ. Babes, Bolyai, Informatica, Vol.LII, No.1, (2007), pp.89-96.

[13] F. Wang, "Improving Remote-Sensing Image-Analysis through Fuzzy Information Representation", Photogrammetric Engineering and Re mote Sensing, Vol.56, No.8, (1990), pp.1163-1169.

[14] K. Zanter, Landsat 8 (L8) Data Users Handbook, LSDS-1574 Version. 2.0, Vol.2, (2016)

[15] Directorate of Census Operations Karnataka, District census handbook Uttara Kannada, Census of India, (2014).

[16] J.P. Pascal, Explanatory Booklet on Forest Map of South India: Belgaum-Dharwar-Panaji, Shimoga, Mercara-Mysore, Institut Francais de Pondichery: Travaux de la Section Scientifique et Technique. Hors Serie N 18, (1986).

[17] USGS, Earthexplorer, https://earthexplorer.usgs.gov/

[18] P.H. Swain, S.M. Davis, Remote Sensing: The Quantitative Approach, McGraw-Hill, pp.166-174, (1978).

[19] J. A. Richards, X. Jia, Remote Sensing Digital Image Analysis- An Introduction, Springer, (2006).

[20] L. A. Zadeh, "Fuzzy sets", Information and Control, Vol.8, No.3, (1965), pp.338-353.

[21] R. Pouncey, K. Swanson, K. Hart, ERDAS Field Guide, ERDAS Inc. (1999).

[22] R.G. Congalton, "A review of assessing the accuracy of classifications of remotely sensed data", Remote Sensing of Environment, Vol.37, No.1, (1991), pp.35-46. 